\title{
Influence of $\omega-3$ polyunsaturated fatty acids on the structure of immunocompetent cell membranes and the parameters of cellular and humoral immunity in children with rheumatoid arthritis
}

\author{
NATALIIA YAREMA ${ }^{1, A, ~ B, ~ D-F}$, OLGA FEDORTSIV², A, D, BOHDAN PALASIUK, C, E \\ ${ }^{1}$ Department of Child Diseases and Child Surgery, I. Horbachevsky Ternopil State Medical University, \\ Ternopil, Ukraine \\ ${ }^{2}$ Higher School of the National Economy in Kutno, Poland \\ ${ }^{3}$ Medical Physics Department, I. Horbachevsky Ternopil State Medical University, Ternopil, Ukraine
}

A - Study Design, B - Data Collection, C - Statistical Analysis, D - Data Interpretation, E - Manuscript Preparation, F - Literature Search, G - Funds Collection

Summary Background. $\omega-3$ polyunsaturated fatty acids ( $\omega-3$ PUFA) in treatment of inflammations were presented in many clinical studies. Although inflammatory responses are carried out by a wide spectrum of cells, CD4 T-cells are crucial in the etiology of many chronic inflammatory diseases, e.g. inflammatory bowel disease, systemic lupus erythematosus, psoriatic arthritis and obesity. In light of recent concerns over the safety of non-steroidal anti-inflammatory drugs (NSAIDs), the alternatives, such as bioactive agents, are becoming more attractive. In order for these agents to be recognized by mainstream medicine, the mechanisms of anti-inflammatory effect of bioactive agents, such as $\omega-3$ PUFA, should be fully revealed.

Objectives. The aim of the research was to evaluate the influence of $\omega$-3 PUFA on immunocompetent cell membranes and the parameters of cellular and humoral immunity in children with rheumatoid arthritis (RA).

Material and methods. With the consent of their parents, 53 children hospitalized with RA and 15 healthy children 5-16 years of age were enrolled in the research. The children were randomized into 2 groups: the first group of the examined patients underwent backbone therapy, the second - backbone therapy with $\omega$-3 PUFA. Immunity parameters (CD3, CD4, CD8, CD16, CD22, IgA, IgM, IgG, IL-1, IL-4, IL-6, amount of circulating immune complexes (CIC) in blood serum) were evaluated, as well as the metastructure of lymphocytes was investigated morphologically at the beginning of the investigation and after treatment.

Results and discussion. A more apparent improvement of immune parameters was demonstrated in the groups undergoing backbone therapy supplemented with $\omega-3$ PUFA. Statistically significant differences between the parameters of the two treatment methods were verified: $\operatorname{lgA}(2.27 \pm 0.14)$ and $(1.96 \pm 0.02) \mathrm{g} / \mathrm{I}(p \leq 0.01), \operatorname{lgM}(1.94 \pm 0.11)$ and $(1.64 \pm 0.05) \mathrm{g} / \mathrm{I}(p \leq 0.01), \mathrm{CD} 8(17.85 \pm 0.33)$ and $(19.33$ $\pm 0.16) \%(p \leq 0.001), \mathrm{CD} 22(10.9 \pm 0.2)$ and $(10.42 \pm 0.09) \%(p \leq 0.01), \mathrm{IL}-1(118.55 \pm 5.22)$ and $(97.69 \pm 3.14) \mathrm{pg} / \mathrm{ml}(p \leq 0.001), \mathrm{IL}-4$ $(113.05 \pm 5.15)$ and $(92.63 \pm 2.78) \mathrm{pg} / \mathrm{ml}(p \leq 0.001)$. $\omega-3$ PUFA stabilize lymphocyte cell membranes and activate the organoid level of intracellular regeneration.

Conclusions. $\omega$-3 PUFA increase the effect of backbone therapy and has proven to be an important supplement in the diet therapy of children with RA.

Key words: rheumatoid arthritis (RA), fatty acids, immune system, cel membrane, child.

Yarema N, Fedortsiv O, Palasiuk B. Influence of $\omega-3$ polyunsaturated fatty acids on the structure of immunocompetent cell membranes and the parameters of cellular and humoral immunity in children with rheumatoid arthritis. Fam Med Prim Care Rev 2018; 20(1): 78-82, doi: https://doi.org/10.5114/fmpcr.2018.73707.

\section{Background}

Rheumatoid arthritis (RA) is a chronic immune-mediated disease caused by monocyte/macrophage activation that leads to local inflammation and destruction of articular cartilage and bone due to proteolytic enzymes and prostaglandin production; the development and course of the disease depends on T-helpers [1]. B-lymphocytic activation of synovium inflammation followed by autoantibody production is crucial in rheumatoid development. The imbalance between the production of pro-inflammatory and anti-inflammatory cytokines, when the production of the former exceeds the latter, is significant in RA pathogenesis [2]. Cytokine cascade combination with humoral dysimmunity and activation of free-radical oxidation increase abnormal changes in joint connective tissue and contribute to their chronization [3].
Numerous theoretical research during the past 2-3 decades has advanced in the accumulation of evidence of various effects of $\omega-3$ PUFA in adult patients. Their anti-inflammatory, antiplatelet, immunomodulatory and antiarrhythmic effects have been established [4, 5].

Taking into account current ideas about the significance of $\omega-3$ PUFA in regulation of an immune response, inflammatory reactions, hemostasis and other key physiological functions, special attention should be paid to the possible deficiency of these nutrients.

It has also been proven that in long chain PUFA metabolism, the lipid mediators of inflammation, such as prostaglandins, leukotrienes and thromboxanes, are formed. At the same time, various types of lipid mediators that differ in their activity are formed of different types of PUFA [6]. Prostaglandin PGE2 and LTB4 are products of arachidonic acid (AA), and other $\omega-6$ PUFA 
may be modified into AA. AA is the main omega-6 PUFA, which can be metabolized into PGE2 and LTB4 by cyclooxygenase (COX) or lipoxygenase (LOX). LTB4 is formed of AA by 5-lipoxygenase and is a chemoattractant and activator of neutrophils; thus, they are crucial for inflammatory arthritis development. Considering that decreased $A A$ production causes reduction in PGE2 and LTB4, AA concentration is significant in the synthesis of both PGE2 and LTB4. Metabolites of PUFA of $\omega-6$ type - leukotrienes of the $4^{\text {th }}$ series, undertake inflammatory activity, whereas, in contrast, PUFA of $\omega-3$ type - leukotrienes of the $5^{\text {th }}$ series, undertake anti-inflammatory activity. Inhibiting the lypooxygenating method of eicosatetraenoic acid transformation and decreasing the formation of leukotrienes of the $4^{\text {th }}$ series - inflammatory inducers, chemotaxis and leukocyte adhesion, leukotrienes of the $5^{\text {th }}$ series, have a stimulating effect on T-cell immunity and phagocytosis [7]. Thus, the inhibition of arachdonic acid metabolites, especially their product by the 5-lipoxygenase pathway, leukotriene B4, is considered to be the most important mechanism of the $\omega$-3 PUFA effect.

Taking into account pathophysiological peculiarities of the rheumatoid process and the significance of $\omega$-3 PUFA in immune cell membrane functioning, the clinical efficacy of medicines containing these $\omega-3$ PUFA in treating children was studied.

\section{Objectives}

The aim of the research was to evaluate the influence of $\omega-3$ PUFA on immunocompetent cell membranes and the parameters of cellular and humoral immunity in children suffering from RA.

\section{Material and methods}

Informed consent was the criteria for the patients' enrolment in the research. Diagnostic data was determined according to the criteria of the American College of Rheumatology (ACR) on grounds of the evidence in children of morning stiffness (lasting not less than 1 hour), arthritis of three and more joints or hand joints, symmetric arthritis, rheumatoid nodules, rheumatoid factor in patients' serum, radiologic abnormality; children's age not over 16 years old. Rheumatologic evaluation of the children with RA comprised clinical examination, assessment of disease activity rate by means of the Disease Activity Score (DAS 28) that involved 28 joints (tenderness and swelling) by visual analogue scale (VAS) ranging $0-10$ [8].

Absence of clinically significant concomitant diseases, stable therapy with non-steroidal anti-inflammatory drugs and corticosteroids, duration of study not less than 3 months were the criteria for enrolment of children with RA in the research.

After consent from the parents, 53 children suffering from RA and 15 healthy children (control group) 5-16 year of age were examined. The children were randomized into 2 groups: $1^{\text {st }}$ group - 20 children who underwent backbone therapy; $2^{\text {nd }}$ group - backbone therapy and Epadol (Kyiv Vitamin Factory): 1 capsule contained $1,000 \mathrm{mg}$ of $\omega-3$ acids consisting of $300 \mathrm{mg}$ of eicosapentaenoic acid (C20:5 $\omega-3)$ and $200 \mathrm{mg}$ of docosahexaenoic acid (C22:6 $\omega-3), 498 \mathrm{mg}$ of other fatty acids and $2 \mathrm{mg}$ of $d$ - $\alpha$-tocopherol: pre-school-aged children took a single dose of $0.5 \mathrm{~g}$ twice a day; primary school-aged children $-0.5 \mathrm{~g}$ three times a day; secondary school-aged children - $1 \mathrm{~g}$ three times a day (after a meal) over three months of the hospital treatment.

All children underwent a complete clinical, laboratory and instrumental examination on admission. The examination was conducted twice: before and after the course of treatment. To evaluate the immunity status, IL-1, IL-4, IL- 6 and the T-cell subset (CD3, CD4, CD8, CD16, CD22) in peripheral blood were determined by means of flow cytofluorometry according to Remy B.G. Thoenes, U. Wahl. Functional activity of B-lymphocytes was determined by the concentration of immune serum globulins of the main classes ( $M, G, A$ ) according to $G$. Mancini et al. [9]. The amount of immune complexes in blood serum was estimated according to the Y.I. Khotkova method.

Morphological examination of leucocytes: $4 \mathrm{ml}$ of blood from the cubital vein of children with RA was centrifuged and further set in a $2.5 \%$ glutaraldehyde solution and phosphate buffer $\mathrm{pH} 7.2-7.4$ and fixed by $1 \%$ solution of osmic acid. After this, the material was dewatered in spirits and acetone, painted with a $2 \%$ uranyl acetate solution and embedded in an epoxy resin mixture. Ultrathin sections were made by means of ultra-microscope UMTP-7, counterstained with lead citrate according to Reynold, studied and photo-recorded using an EMB-100 LM electronic microscope. Microscopic slides were analyzed by light microscopy under 1,500-fold magnification.

Ethical protocols approved by the ethical committee of I. Horbachevsky Ternopil State Medical University (Ukraine) were introduced to all children.

\section{Statistical analysis}

Statistical processing of data was carried out using the SPSS Statistics 21.0 program. Statistical significance was estimated by Mann-Whitney and Wilcoxon non-parametric tests. In the case of normal index distribution, the statistical differences were determined by the Student $t$-test. The significance level was reliable at $p<0.05$.

\section{Results}

Before treatment of the RA children, significant changes in the cellular component of the immune system - decrease in bulk population of CD3-leucocytes, were observed. This took place due to a decrease of CD8-suppressors (16.50 $\pm 0.51 \%)$ and CD4-helpers (35.60 $\pm 0.56 \%)$. It was established that the CD4-helpers activate proliferation, differentiation and the cytotoxic effects of killer-cells and stimulate functional activity of B-lymphocytes and macrophages as a consequence of production of specific and non-specific helper factors.

On the basis of the decrease in the level of CD-8 suppressors, an increase of CD-22 (B-lymphocytes) up to $12.55 \pm 0.32 \%$ in comparison with the control group $(9.40 \pm 0.25 \%)$ was observed. Their proliferation led to production of plasmatic cells, which synthesize autoantibodies (Table 1).

A significant increase of immunoglobulins of the main classes: IgA (3.18 $\pm 0.12 \mathrm{~g} / \mathrm{l})$, IgM $(2.57 \pm 0.16 \mathrm{~g} / \mathrm{l})$, IgG $(14.63 \pm 0.43$ $\mathrm{g} / \mathrm{l})$, proved the activation of the humoral component of the immune system in the children. A CIC increase (147.5 $\pm 4.11 \mathrm{RU})$ evidenced the immunopathological response in children. The $\mathrm{CIC}$ level was dependent upon process activity, presence of visceral manifestations and previous treatment with hormonal and cytostatic drugs.

A significant increase of $\mathrm{ClC}$ was evidenced by disease onset, which lasted up to 1 year, as well as on-disease recurrences with a 3-year duration of the disease. A decrease in the $\mathrm{CIC}$ amount ( $83 \pm 1.20 \mathrm{RU}$ and $56 \pm 10 \mathrm{RU}$, respectively) was proven in the case of disease duration up to 3 years and visceral manifestations in the RA children.

The imbalance between the levels of anti-inflammatory cytokines IL-1, IL- 6 and anti-inflammatory IL-4 proved to produce a high inflammatory reaction in the RA children. A decreased activity of $C D 4, C D 8$, immunoregulatory index, activation of the humoral component of the immune system with a $\mathrm{CIC}$ increase, imbalance of the system of cytokines are essential in the development of inflammation and destructive pathology of joints in cases of RA.

An upward trend of the investigated rates of both courses of therapy used was evidenced by comparing the efficacy of the treatment of RA children. However, immunological status indices proved the prevailing positive effect of therapy supplemented with $\omega-3$ PUFA. Thus, at the end of the treatment course, an in- 


\begin{tabular}{|c|c|c|c|c|c|c|c|c|}
\hline \multirow{3}{*}{$\begin{array}{l}\text { Researched } \\
\text { index }\end{array}$} & \multirow{3}{*}{$\begin{array}{l}\text { Control } \\
\text { group, } \\
n=15\end{array}$} & \multicolumn{4}{|c|}{ Children with RA } & \multirow[t]{3}{*}{$p_{1}$} & \multirow[t]{3}{*}{$p_{2}$} & \multirow[t]{3}{*}{$p_{3}$} \\
\hline & & \multicolumn{2}{|c|}{ backbone therapy, $n=20$} & \multicolumn{2}{|c|}{ modified therapy, $n=33$} & & & \\
\hline & & $\begin{array}{l}\text { before treat- } \\
\text { ment }\end{array}$ & after treatment & $\begin{array}{l}\text { before treat- } \\
\text { ment }\end{array}$ & after treatment & & & \\
\hline $\lg A, g / l$ & $1.90 \pm 0.12$ & $3.18 \pm 0.12$ & $2.27 \pm 0.14$ & $2.96 \pm 0.08$ & $1.96 \pm 0.02$ & $\leq 0.001$ & $\leq 0.001$ & $\leq 0.01$ \\
\hline $\operatorname{lgM}, \mathrm{g} / \mathrm{I}$ & $1.43 \pm 0.22$ & $2.57 \pm 0.16$ & $1.94 \pm 0.11$ & $2.55 \pm 0.14$ & $1.64 \pm 0.05$ & $\leq 0.001$ & $\leq 0.001$ & $\leq 0.01$ \\
\hline $\lg G, g / I$ & $10.02 \pm 0.54$ & $14.63 \pm 0.43$ & $12.10 \pm 2.11$ & $13.38 \pm 0.23$ & $10.97 \pm 0.11$ & $>0.05$ & $\leq 0.001$ & $>0.05$ \\
\hline $\mathrm{CD} 3, \%$ & $65.12 \pm 0.24$ & $60.45 \pm 1.02$ & $65.55 \pm 0.87$ & $61.48 \pm 0.72$ & $66.90 \pm 0.44$ & $\leq 0.001$ & $\leq 0.001$ & $>0.05$ \\
\hline CD4, \% & $39.24 \pm 2.12$ & $35.60 \pm 0.56$ & $35.95 \pm 0.37$ & $34.45 \pm 0.45$ & $36.93 \pm 0.18$ & $>0.05$ & $\leq 0.001$ & $\leq 0.05$ \\
\hline CD8, \% & $19.81 \pm 1.20$ & $16.50 \pm 0.51$ & $17.85 \pm 0.33$ & $16.12 \pm 0.30$ & $19.33 \pm 0.16$ & $>0.05$ & $\leq 0.001$ & $\leq 0.001$ \\
\hline CD4/CD8, \% & $1.82 \pm 0.04$ & $2.19 \pm 0.07$ & $2.08 \pm 0.04$ & $2.16 \pm 0.05$ & $1.85 \pm 0.02$ & $>0.05$ & $\leq 0.001$ & $\leq 0.001$ \\
\hline CD16, \% & $14.73 \pm 0.54$ & $12.65 \pm 0.25$ & $12.70 \pm 0.16$ & $12.87 \pm 0.20$ & $12.27 \pm 0.18$ & $>0.05$ & $>0.05$ & $\leq 0.05$ \\
\hline CD22, \% & $9.40 \pm 0.25$ & $12.55 \pm 0.32$ & $10.90 \pm 0.20$ & $13.03 \pm 0.13$ & $10.42 \pm 0.09$ & $\leq 0.001$ & $\leq 0.001$ & $\leq 0.01$ \\
\hline IL-1, pg/ml & $37.91 \pm 4.96$ & $145.40 \pm 4.57$ & $118.55 \pm 5.22$ & $139.12 \pm 3.77$ & $97.69 \pm 3.14$ & $\leq 0.001$ & $\leq 0.001$ & $\leq 0.001$ \\
\hline $\mathrm{IL}-4, \mathrm{pg} / \mathrm{ml}$ & $15.51 \pm 0.02$ & $126.80 \pm 5.60$ & $113.05 \pm 5.15$ & $125.30 \pm 2.58$ & $92.63 \pm 2.78$ & $\leq 0.05$ & $\leq 0.001$ & $\leq 0.001$ \\
\hline IL-6, pg/ml & $7.69 \pm 3.41$ & $79.85 \pm 3.04$ & $64.40 \pm 3.79$ & $77.84 \pm 1.08$ & $64.30 \pm 1.09$ & $\leq 0.001$ & $\leq 0.001$ & $>0.05$ \\
\hline CIC c.u. & $60.54 \pm 1.25$ & $147.50 \pm 4.11$ & $130.70 \pm 13.6$ & $143.42 \pm 9.22$ & $105.00 \pm 7.05$ & $>0.05$ & $\leq 0.001$ & $\leq 0.05$ \\
\hline
\end{tabular}

$p_{1}$ - significant differences of parameters before and after backbone therapy; $p_{2}$ - significant differences of parameters before and after modified therapy; $p_{3}$ - significant differences of parameters before and after backbone and modified therapy in children with RA.

crease of total CD3-lymphocytes $(p<0.05)$ was observed in the $1^{\text {st }}$ group. The levels of CD4-helpers $(35.95 \pm 0.37 \%)$ and CD8-suppressors $(17.85 \pm 0.33 \%)$ in the children of this group were below the normal level, and stabilization of the immunoregulatory index did not take place. The level of B-lymphocytes (10.9 $\pm 0.2 \%$ ) was high, but their functional activity was partially restricted. This was proved by the dynamics of immunoglobulin, as its content stayed within a normal level after treatment.

Therefore, dysfunction of the T-component of the immune response and activation of humoral responses and immunopathological processes occurred. A tendency towards stabilization of immunological parameters was evidenced on the basis of conventional drug treatment only (Table 1).

Edema and sharp cytoplasm clearing and destruction of rough endoplasmic reticulum with vacuolation was observed during histological processing of the lymphocyte metastructure of children with RA. There was no evidence of crista in the mitochondria, and the matrix was light and vacuolated. The nucleus was of irregular shape because of intussusceptions of various depths with abruptly condensed chromatin, and the perinuclear space was widened with vacuolation areas.

In cases of high activity of inflammatory processes in children with RA, myelin-like structures generated by nucleus membranes and mitochondria were revealed. The central part of the cleared cytoplasm of these lymphocytes was occupied by a round-shaped nucleus with light intussusceptions. The external part of the nucleolonema formed myelin-like structures. Rounded mitochondria had a granular matrix of increased osmiophilia, and some of them were transformed into myelin-like bodies. In the nucleus intussusception area, karyolemma was destroyed and cytoplasm "portion" necrosis developed.

The most significant changes of leukemic membrane components accompanied by organelle alteration and formation of myelin-like structures were determined in cases of high activity of inflammatory processes in children with a joint-visceral form of RA.

Thus, during histological processing of the lymphocyte metastructure of children with RA, edema and sharp cytoplasm clearing and destruction of rough endoplasmic reticulum with vacuolation were evidenced (Figure 1). There was no evidence of crista in the mitochondria, and the matrix was light and vacuolated.

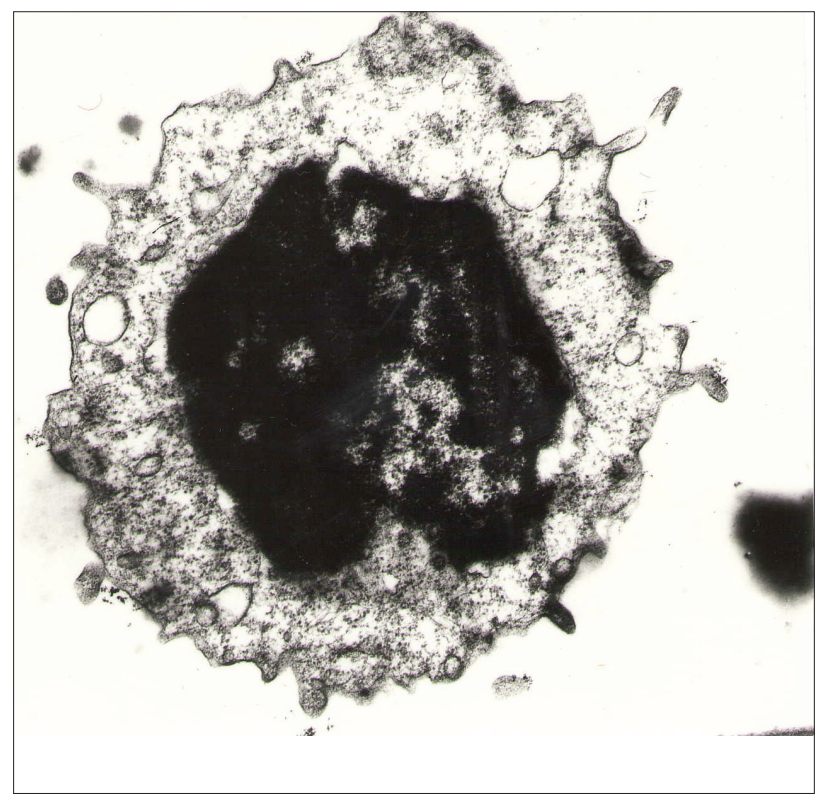

Figure 1. Submicroscopic structure of blood lymphocyte of children with RA. ME magn. $\times 1,500$

A study of the submicroscopic structure of blood lymphocytes in the children with RA after treatment proved that along with normal morphology of the leukemics, there were cells with dominating hyperplastic processes of the nucleus and organelles in their cytoplasm. The perinuclear space was narrow, the external part of karyolemma was densely spread with ribosomes, and multiple pores were observed. Short canaliculi of rough endoplasmic reticulum, multiple ribosomes and polysomes were found. Mitochondria were multiple, predominantly of a round shape with a moderate osmiophilic matrix and sharply contoured crypts. There were single organelles with a vacuolated matrix and shortened crista among them.

Therefore, the treatment obviously stabilized cell membranes and activated the organoid level of intracellular regeneration (Figure 2). 


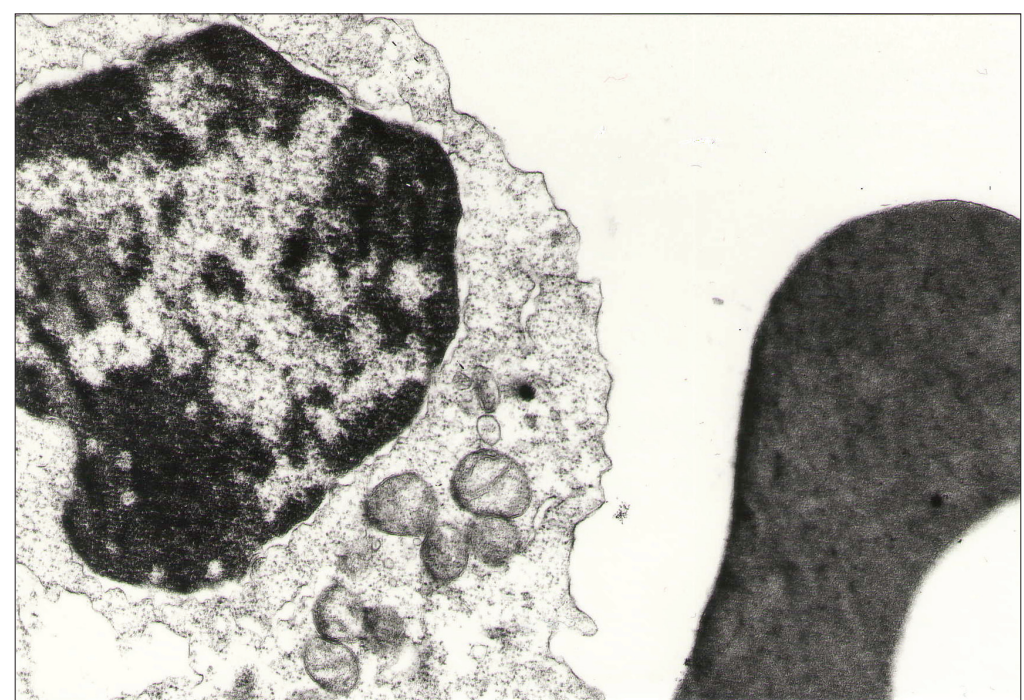

Figure 2. Submicroscopic structure of blood lymphocyte affected by treatment in the case of high RA activity. ME magn. $x 1,500$

\section{Discussion}

Several researchers studied the positive effects of $\omega$-3 PUFA dietary supplements in adult patients with RA on tender joint count, duration of morning stiffness and, less frequently, handgrip strength, Ritchie index, patient global assessment, medical global assessment, patient and doctor pain intensity evaluation [10-12]. Meta-analysis proving the efficacy of $\omega$-3 PUFA in treatment of children with RA was carried out by Lee, Bae and Song. 10 RCTs involving 183 RA children and 187 placebo-treated RA controls comprised this meta-analysis [13]. The analysis proved that $\omega-3$ PUFA in the therapy of RA children decreased non-steroidal anti-inflammatory drug (NSAID) consumption, tender joint count, joint swelling and duration of morning stiffness [4].

However, the influence of $\omega-3$ PUFA on the immune system of children with RA, which is crucial for the pathogenesis of this seriously disabling disease, has not been adequately studied.

The examined children with RA had decreased levels of CD4, CD8, hypoactivity, decreased immunoregulatory index, activation of the humoral component of the immune system with increased $\mathrm{CIC}$, imbalance of the system of cytokines and ultrastructural alteration of lymphocytes. The research proved that T-cells are activated in the peripheral blood and cross the endothelial cell wall and migrate into the joints. In joint synovia, T-cells are reactivated by cross-reactive antigens and enlarge clonally. Clonally enlarged T-cells accumulate in the damaged joints and secrete proinflammatory cytokines that captivate and activate other cells, such as monocytes and macrophages [14]. The established decrease of the (CD4/CD8) immunoregulatory index can prove the introduction of a lipid metabolism decompensation mechanism into T-lymphocyte biomembranes.

In our opinion, a reliable $\mathrm{CIC}$ increase is caused by the development of secondary immunodeficiency and $\mathrm{CIC}$ seeding at target organs, leading to visceral damage.

Introduction of a $\omega-3$ PUFA drug into the comprehensive treatment of children with RA positively affects the main pathogenetic components of the pathologic process. In our opinion, this possible immunomodulatory effect is due to the ability of $\omega-3$ PUFA to decrease synthesis of immunoregulatory and anti-inflammatory cytokines by mononuclear cells, as well as the possibility to replace arachidic acid in the phospholipid membranes of immunocompetent cells on a competitive base. The research proved that $\omega-3$ PUFA compete with the inhibition of PGE2 and LTB4 production, which consecutively inhibits the activation of NF-KB and thus the release of inflammatory cytokines IL-1 $\beta$ and TNF- $\alpha$ [15].
In case of a high inflammation activity in children with RA, myelin-like structures generated by nucleus membranes and mitochondria are observed in a leucocyte photograph. These are structural proof of a free radical effect as the result of the activation of membrane lipid peroxidation; they are formed of released phospholipids by torsion of extended cytoplasmic tails or microtubules. The presence of these structures in mitochondria is evidence of cell exhaustion (decreased glutathione, natural structural antioxidants: vitamin $\mathrm{E}$ and cholesterol) and the damaging effect of lipid peroxidation. In addition, the functional capabilities of immunocompetent cells are determined by their intracellular metabolism: free-radical oxidation and formation of reactive oxygen intermediates are crucial. Activation of free-radical oxidation, protease system and "primary" and gastrointestinal digestion disorders induced by treatment are pathogenetic factors causing multicomponent damage of membrane structures, even at early stages of RA. Thus, the criteria of pathologic process severity and therapeutic intervention effectiveness are the disorders of microviscosity and membrane structures penetration but not the increased generation of reactive oxygen intermediates. The results of our research conform to the results of other studies comprising data on the positive effect of $\omega-3$ PUFA in comprehensive treatment of inflammatory diseases. Above all, Hou and McMurray showed that introduction of $\omega-3$ PUFA changed the biochemical and biophysical properties of the immune system of the $\omega-3$ plasma-membrane, which contributed to modulation of intracellular mechanisms and remission of autoimmune and chronic inflammatory diseases [16].

The treatment supplemented with $\omega-3$ PUFA resulted in stabilization of membrane components and restoration of the leukocyte ultrastructure. Therefore, complex therapy, as well as $\omega$-3 PUFA, causes a decrease of inflammatory material synthesis and has a membrane stabilizing and immune modelling effect due to replacement of arachidic acid with $\omega-3$ PUFA in phospholipid cells on a competitive basis. $\omega$-3 PUFA influence on the core of the RA pathogenetic area sufficiently increases the effectiveness of backbone therapy and prolongs clinical remission in children. Moreover, having no adverse effect is the most definitive factor in pediatric practice, which is the most important advantage of this form of treatment performed on children.

\section{Conclusions}

The following disorders were diagnosed in the immune status of children with RA: decrease of CD4 and CD8, CD4/ /CD8 activity, activation of humoral component, increase in CIC amount, imbalance of the cytokines system. The use of both 
regimens had a positive effect on the immune status of children with RA, but the most significant differences of parameters (IgA, IgM, CD8, CD4/CD8, CD22, IL-1, IL-4, CIC) were evidenced in the group of children who underwent backbone therapy supplemented with $\omega-3$ PUFA $(p \leq 0.05)$.
The structure of cell membranes defines the functional capabilities of immunocompetent cells and is crucial for pathogenesis of this seriously disabling disease. The functional membrane structure of immunocompetent cells can be improved with introduction of $\omega$-3 PUFA into RA comprehensive treatment.

Source of funding: This work was funded by the authors' own resources.

Conflict of interest: The authors declare no conflict of interests.

\section{References}

1. Langford RM. Pain management today - what have we learned? Clin Rheumatol 2006; 25(Suppl. 1): S2-S8.

2. Chen M, Lam BK, Kanaoka Y, et al. Neutrophil-derived leukotriene B4 is required for inflammatory arthritis. J Exp Med 2006; 203(4): 837-842.

3. Cleland LG, James MJ, Proudman SM. Fish oil: what the prescriber needs to know. Arthritis Res Ther 2006; 8(1): 202.

4. Calder PC. n-3 polyunsaturated fatty acids, inflammation, and inflammatory diseases. Am J Clin Nutr 2006; 83(6 Suppl.): 1505S-1519S.

5. Goldberg RJ, Katz J. A meta-analysis of the analgesic effects of omega-3 polyunsaturated fatty acid supplementation for inflammatory joint pain. Pain 2007; 129(1-2): 210-223.

6. World Health Organisation. Population nutrient intake goals for preventing diet-related chronic diseases [cited 06.11.2014]. Available from URL: http://www.who.int/nutrition/topics/5_population_nutrient/en/index13.html.

7. Barros R, Moreira A, Fonseca J, et al. Dietary intake of alpha-linolenic acid and low ratio of n-6:n-3 PUFA are associated with decreased exhaled NO and improved asthma control. BrJ Nutr 2011; 106(3): 441-450.

8. Fransen J, van Riel PL. The disease activity score and the EULAR response criteria. Clin Exp Rheumatol 2005; 23(5 Suppl. 39): S93-S99.

9. Mancini G, Carbonara AO, Heremans JF. Immunochemical quantitation of antigens by single radial immunodiffusion. Immunochemistry 1965; 2(3): 235-254.

10. Yarema NM. $\omega-3$ polyunsaturated fatty acids use for optimization of children inflammatory joints diseases treatment. Voprosy pitaniia 2017; 2: 84-90.

11. Casula M, Soranna D, Catapano AL, et al. Long-term effect of high dose omega-3 fatty acid supplementation for secondary prevention of cardiovascular outcomes: a meta-analysis of randomized, placebo controlled trials [corrected]. Atheroscler Supp/ 2013; 14(2): 243-251.

12. Galarraga B, Ho M, Youssef HM, et al. Cod liver oil (n-3 fatty acids) as a non-steroidal anti-inflammatory drug sparing agent in rheumatoid arthritis. Rheumatology (Oxford) 2008; 47(5): 665-669.

13. Lee YH, Bae SC, Song GG. Omega-3 polyunsaturated fatty acids and the treatment of rheumatoid arthritis: a meta-analysis. Arch Med Res 2012; 43(5): 356-362.

14. VanderBorght $A$, Geusens $P$, Raus J, et al. The autoimmune pathogenesis of rheumatoid arthritis: role of autoreactive T cells and new immunotherapies. Semin Arthritis Rheum 2001; 31(3): 160-175.

15. McCann ME, Moore JN, Carrick JB et al. Effect of intravenous infusion of omega-3 and omega-6 lipid emulsions on equine monocyte fatty acid composition and inflammatory mediator production in vitro. Shock 2000; 14(2): 222-228.

16. Hou TY, McMurray DN, Chapkin RS. Omega-3 fatty acids, lipid rafts, and T cell signaling. Eur J Pharmacol 2016; 785: 2-9, doi: 10.1016/j. ejphar.2015.03.091.

Tables: 1

Figures: 2

References: 16

Received: 11.02.2017

Reviewed: 12.04 .2017

Accepted: 04.10.2017

Address for correspondence:

Nataliia Yarema, MD, PhD

Department of Children Diseases and Children Surgery

I. Horbachevsky Ternopil State Medical University

2 Sakharova Str.

Ternopil

46001

Ukraine

Tel.: +380-979726307, 380-352-250000

E-mail: yarema_nm@tdmu.edu.ua 